\title{
Estimación de parámetros genéticos para caracteres de crecimiento en bovinos criollos Romosinuano
}

\author{
Estimation of genetic parameters for Growth traits in Romosinuano creole Cattle
}

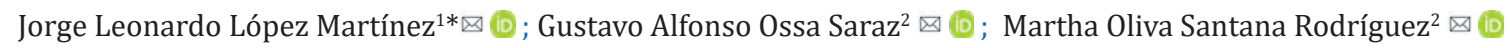

\begin{abstract}
${ }^{1}$ Universidad Politécnica de Valencia, Valencia, España.
${ }^{2}$ Corporación Colombiana de Investigación Agropecuaria - Agrosavia, Centro de Investigación Turipaná, Cereté, Córdoba, Colombia.

*Correspondencia: jollopezma@unal.edu.co
\end{abstract}

\section{RESUMEN}

Objetivo. Estimar la heredabilidad y las correlaciones de tres caracteres relacionados con el crecimiento en bovinos criollos Romosinuano, y construir un índice de selección (IS). Materiales y métodos. Se analizó una base de datos conformada por 4079 registros de peso al nacimiento (PN), al destete (PD) y a los 16 meses de edad (P16m), obtenidos en el periodo 1943-2018, proveniente del hato de ganado criollo colombiano Romosinuano del centro de investigación Turipaná. Se estimaron tanto los valores de heredabilidad para cada carácter como sus correlaciones, mediante el modelo animal multivariado; los valores genéticos (VG) y su relación usando el análisis de componentes principales. Resultados. Las correlaciones genéticas entre PN y PD, PN y P16m, y PD y P16m fueron de magnitud moderada a alta y positivas, lo que indica una asociación genética favorable entre estos caracteres. Los VG estandarizados para PN, PD y P16m exhibieron una alta asociación con el primer componente principal. Las estimaciones de heredabilidad total para PN, PD y P16m fueron igual a $0.18 \pm 0.04,0.16 \pm 0.04$ y $0.13 \pm 0.04$, respectivamente. Conclusiones. Los caracteres PN, PD y P16m responderán lentamente a la selección, sin embargo, podría mejorarse mediante la construcción de IS basados en los componentes principales.

Palabras clave: Análisis multivariado; componentes principales; correlaciones genéticas; ganado criollo; heredabilidad; índices de selección; Romosinuano; valores genéticos.

\begin{abstract}
Objective. Estimate the heritability and correlations of three growth-related traits in Romosinuano Creole cattle, and to construct a selection index (IS). Materials and methods. A database made up of 4079 records of weight at birth (PN), weaning (PD) and at 16 months of age (P16m), obtained in the period 1943-2018, from the Turipaná research center herd of Romosinuano Colombian creole cattle was analyzed. Both the heritability values for each character and their correlations were estimated using the multivariate animal model; genetic values (VG) and their relationship using principal component analysis. Results. Genetic correlations between PN and PD, PN and P16m, and PD and P16m were moderate to high magnitude and positive, indicating a favorable genetic association between these traits. The standardized VGs for PN, PD and P16m exhibited a high association with the first principal component. The estimates of total heritability for PN, PD and P16m were equal to $0.18 \pm 0.04,0.16 \pm 0.04$ and $0.13 \pm 0.04$, respectively. Conclusions. suggesting that these characters will respond slowly to selection, however, it could be improved by constructing IS based on the main components.
\end{abstract}

Keywords: Creole cattle; genetic correlations; genetic values; heritability; main components; multivariate analysis; Romosinuano; selection index. 


\section{INTRODUCCION}

El crecimiento está determinado por los efectos genéticos y/o ambientales los cuales inciden de modo diferente durante el tiempo (1). Tanto el proceso fisiológico de crecimiento como el de desarrollo son de gran relevancia en producción de carne y su eficiencia determina gran parte del proceso productivo, el cual juega un papel protagónico en la seguridad alimentaria al ser la carne un alimento de alto valor nutricional.

En Colombia la producción de carne bovina se basa en genotipos de continua introducción con predominio de las razas cebuinas. No obstante, al poseer la mayor diversidad de bovinos localmente adaptados en América, está en la capacidad de desarrollar una producción bovina sostenible, con base en sus propios recursos y de esta manera contribuir a la solución de la creciente demanda en el consumo de este tipo de alimento, considerando que las otras razas no están lo suficientemente adaptadas a las restricciones climáticas y nutricionales que pueden reducir severamente su eficiencia productiva.

La raza Romosinuano presenta gran aptitud para la producción de carne; es una raza criolla originada en el Valle del rio Sinú, Córdoba, Colombia, producto de la diversidad de ambientes que ha soportado, al aislamiento vivido por muchos años, a las distintas razas que han contribuido a su formación y a las diferencias en los criterios selectivos implantados por el hombre. Su población fue numerosa hasta comienzos del Siglo XX, se redujo debido, principalmente, al cruzamiento con el Cebú y en la actualidad se observa un gran interés por esta, por lo que se espera aumente su población.

Las estimaciones de la variación genética y la evaluación de los animales para los caracteres de importancia económica pueden ayudar a mejorar el comportamiento productivo de los rebaños, ya que los animales se seleccionarían de acuerdo con su valor genético (2); para estimar los valores genéticos que permitirán identificar los animales superiores genéticamente puede utilizarse el análisis univariado de los datos de los animales (3).

En ganado Romosinuano, la heredabilidad y las tendencias genéticas de los caracteres peso al nacimiento, peso al destete y peso a los 16 meses del hato del Centro de investigación Turipaná han sido estimados por medio de seis modelos animales mixtos para cada carácter, mediante el análisis univariado (4). No obstante, este análisis presenta limitaciones al no considerar posibles relaciones genéticas entre los caracteres evaluados (5). Por lo tanto, se propuso el análisis multivariado en el cual los caracteres se analizan de forma simultánea, asumiendo que los registros productivos de un mismo individuo son medidas cuya expresión es genéticamente diferente y correlacionada (4), lo cual permite describir los aspectos de la biología de los caracteres los más cerca posible a la realidad, obtener mayor precisión en los valores genéticos (6) y se considera como un método eficiente para clasificar los bovinos de acuerdo a su potencial productivo en programas de mejoramiento genético (5).

Los valores de correlación que derivan del análisis multivariado estudian la variación simultánea entre variables. Desde el punto de vista de caracteres zootécnicos, estas pueden ser fenotípicas, genéticas y ambientales, cuyos valores de correlación pueden variar entre -1 a +1 . En relación a su interpretación, la correlación fenotípica entre dos caracteres sugiere que si un animal está por encima de la media de un carácter a un tiempo determinado esta ventaja se debe de mantener en una edad posterior; la correlación genética indica en que proporción de los genes con respecto a un carácter afectan a otro; en cambio la correlación ambiental indica en que proporción los factores ambientales que afectan a un carácter afectan a los factores ambientales de otro carácter en una edad posterior. Estas correlaciones pueden ser positivas, negativas o nulas dependiendo del valor estimado para cada correlación (7).

De otro lado, la disposición de los valores genéticos y sus relaciones se pueden explorar mediante el análisis de componentes principales. El análisis de componentes principales es una técnica exploratoria que permite ver la relación entre variables de una manera útil. A partir de su uso es posible reducir la información total contenida en un conjunto de datos, en un número menor de variables ortogonales conocidas como componentes principales, con pérdida mínima de información (8). Son escasos los trabajos en donde se haya usado el análisis de componentes principales en las ciencias animales, sin embargo, estos se destacan por identificar caracteres que podrían usarse como criterios de selección $(9$, 10,11) y en la construcción de los denominados índices de selección $(12,13)$.

Teniendo presente las ventajas del método multivariado, se propuso este estudio el cual tiene como objetivo estimar los parámetros genéticos de heredabilidad para el peso al nacimiento, al destete y a los 16 meses de edad en bovinos criollos Romosinuano, y calcular las correlaciones genéticas entre estos pesos, usando un análisis multivariado; así mismo, se pretendió analizar la relación entre los valores genéticos estimados para dichos caracteres por medio de un análisis de componentes principales con el fin de verificar mediante este análisis si es posible usar alguno de los tres caracteres como criterio de selección y proponer la construcción de un índice de selección. 


\section{MATERIALES Y METODOS}

Población de estudio. La información para este estudio proviene de los registros genealógicos y productivos correspondientes al periodo 1943 a 2018, del núcleo de la raza criolla colombiana Romosinuano del sistema general de Banco de Germoplasma de la Nación para la Alimentación y la Agricultura, el cual se encuentra ubicado en el centro de investigación Turipaná (AGROSAVIA), municipio de Cereté, departamento de Córdoba, en la región caribe colombiana; esta se maneja en pastoreo con suplementación mineral y en la época de sequía se suministra ensilaje para compensar el déficit de forraje.

Caracteres estudiados y depuración de los datos. La base de datos comprende el registro informativo de los caracteres peso al nacimiento, peso al destete y peso a los 16 meses, siendo estos dos últimos pesos ajustados a los 240 y 480 días de edad, respectivamente.

El tratamiento y la depuración de los datos obtenidos de los registros se hizo empleando la metodología y paquetes estadísticos utilizados por (4), que consistió en la formación de cuatro clases definidas así: clase 1 (enero a marzo); clase 2 (abril a junio) clase 3 (julio a septiembre) y clase 4 (octubre a diciembre), basados en el mes de nacimiento. A partir de las variables año y época, y teniendo en cuenta la variable sexo (macho o hembra), se formaron los denominados grupos contemporáneos (año - época - sexo). Se analizaron únicamente los animales con padres conocidos, siendo considerados como padres representativos aquellos que tuvieran cinco descendientes en adelante. También se consideraron solo grupos contemporáneos con más de cinco observaciones para cada uno de los caracteres. En la depuración de los datos se utilizaron los paquetes dplyr y tidyr y stringr del software R 3.6.0 (14) especializado en el análisis estadístico de datos y el paquete pedantics del mismo software estadístico.

Un resumen de la estructura de la información luego de editada la información se muestra en la Tabla 1. Cabe mencionar que todos los caracteres tenían el mismo número de observaciones (es decir, los datos estaban equilibrados).

Tabla 1. Estructura de la información para los caracteres de estudio en vacas de la raza Romosinuano.

\begin{tabular}{|c|c|c|c|c|c|}
\hline Caracteres & Número de observaciones & Promedio & Desvío estándar & Valor mínimo & Valor máximo \\
\hline Peso al nacimiento, kg & & 30.33 & 3.61 & 15 & 47 \\
\hline Peso al destete, $\mathrm{kg}$ & 4079 & 165.79 & 34.46 & 62 & 313 \\
\hline Peso a los 16 meses, kg & & 226.67 & 44.38 & 84 & 368 \\
\hline
\end{tabular}

Fuente: Elaboración propia

Análisis estadístico: definición de efectos fijos y aleatorios. Los valores observados para los caracteres productivos (en este caso los caracteres de crecimiento) se deben tanto al efecto de los genes (o efectos aleatorios) como del ambiente (o efectos fijos). Por tanto, es fundamental en el análisis identificar estos posibles efectos con el fin de que se puedan corregir. En este sentido, el criterio de información Bayesiano (BIC) se utilizó para comparar distintos modelos de regresión basado en parsimonia y ajuste, e identificar con el mismo efecto fijos importantes. Para la selección de efectos aleatorios, fueron ajustados distintos modelos univariados para cada uno de los caracteres haciendo uso del software WOMBAT (15), considerando la estructura de efectos fijos del modelo óptimo previamente definido. La selección de los efectos aleatorios se llevó a cabo por medio de la prueba de razón de verosimilitud logarítmica (LRT):

$L R T=2 \times\left(\log \mathrm{L}_{\mathrm{M} 1}-\log \mathrm{L}_{\mathrm{M} 2}\right)$

donde $\log \mathrm{L}_{\mathrm{M} 1}$ corresponde a la verosimilitud logarítmica del modelo más complejo $\left(\mathrm{M}_{1}\right)$ y $\log \mathrm{L}_{\mathrm{M} 2}$ corresponde a la verosimilitud logarítmica del modelo con el menor número de parámetros $\left(\mathrm{M}_{2}\right)$.

Luego, el resultado de la prueba anterior se consideró como un estadístico de prueba, cuya distribución bajo hipótesis nula se asumió como una chi-cuadrado con un grado de libertad. Por lo cual, sobre la base del juego de hipótesis $\mathrm{H}_{0}: \mathrm{M} 1=\mathrm{M} 2$ contra Ha:M1 $\neq \mathrm{M} 2$, si el valor p obtenido era menor a un nivel de significancia de 0.05 , se concluyó que el efecto aleatorio adicional mejoraba significativamente el modelo (16). Por lo contrario, cuando no existía diferencia significativa ( $p>0.05$ ) entre modelos, el modelo con el menor número de parámetros (más parsimonioso) fue elegido como el más apropiado. 
La descripción general de los distintos efectos a incluir en la modelación de acuerdo con los resultados obtenidos se proporciona en la Tabla 2.

Tabla 2. Efectos fijos y aleatorios, y covariables considerados en la modelación de caracteres productivos en bovinos Romosinuano.

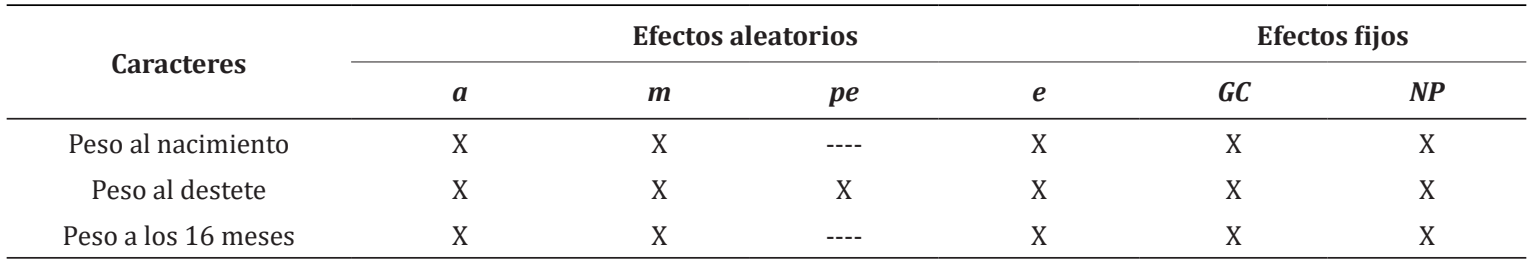

$a$ es el efecto genético directo; $m$ es el efecto genético materno; pe es el efecto ambiental permanente materno; $e$ es el efecto residual; $G C$ es el efecto del grupo contemporáneo; $N P$ es el efecto de número de parto.

Fuente: Elaboración propia

Estimación de parámetros genéticos: modelo multivariado y análisis de componentes principales utilizando los valores genéticos directos. Con base en los efectos fijos y aleatorios de los modelos seleccionados mediante el proceso anterior, fueron estimados los parámetros genéticos para los tres caracteres de crecimiento en bovinos criollos Romosinuano, mediante el método de máxima verosimilitud restringida (REML) para modelos animales multivariados haciendo uso del software WOMBAT (15). El criterio de convergencia para el algoritmo AIREML se estableció en 1x10-12. La matriz de relación incluyó 5215 animales con 1762 madres y 347 padres.

Los modelos multivariados propuestos para los caracteres estudiados se describen a continuación:

$y=X b+Z_{a} a+Z_{m} m+e[1]$

$y=X b+Z_{a} a+Z_{m} m+Z_{p e} p e+e[2]$

donde $y$ es un vector de observaciones para las características de crecimiento (peso al nacimiento, al destete y a los 16 meses); $b, a, m$ y pe son los vectores de efectos fijos (número de parto y grupos contemporáneos), efectos genéticos directos, efectos genéticos maternos y efectos ambientales permanentes maternos, respectivamente; $X, Z_{a}, Z_{m}$ y $Z_{p e}$ son las matrices de incidencia que relacionan los efectos fijos, genéticos directos, genéticos maternos y ambientales permanentes maternos con el vector de observaciones, respectivamente; es el vector de efectos residuales.

El supuesto para los efectos fijos del modelo fue igual a $E[y]=X b$. Los supuestos para los efectos aleatorios del modelo y su estructura de (co) varianza se describen a continuación:

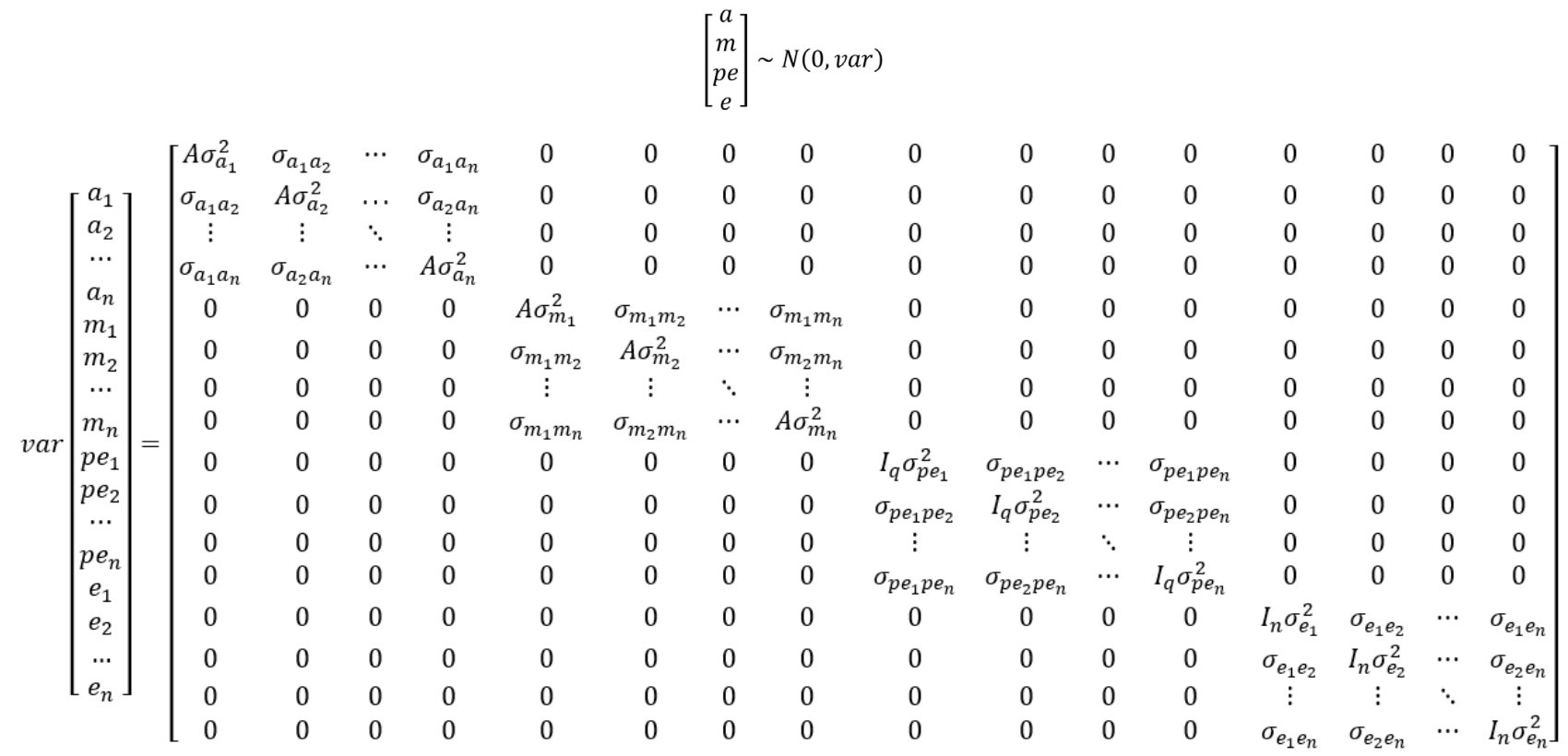


donde $\sigma_{\mathrm{a}_{1}}{ }^{2} \mathrm{a} \sigma_{\mathrm{a}_{\mathrm{n}}}{ }^{2}$ son las varianzas del efecto genético directo, $\sigma_{\mathrm{m}_{1}}{ }^{2} \mathrm{a} \sigma_{\mathrm{m}_{\mathrm{n}}}{ }^{2}$ son las varianzas del efecto genético materno, $\sigma_{\mathrm{pe}}{ }^{2}$ a $\sigma_{p e_{n}}{ }^{2}$ son las varianzas del efecto de ambiente permanente, $\sigma_{e_{1}}{ }^{2}$ a $\sigma_{e_{n}}{ }^{2}$ son las varianzas del efecto residual, $\sigma_{a_{1} a_{2}}$ a $\sigma_{a_{n} a_{n-1}}$ es la covarianza genética directa entre los caracteres, $\sigma_{m_{1} m_{2}}$ a $\sigma_{m_{n}} m_{n-1}$ es la covarianza genética materna entre los caracteres, $\sigma_{\mathrm{pe}_{1}} \mathrm{p}_{\mathrm{e}_{2}}$ a $\sigma_{\mathrm{pe}_{\mathrm{n}}} \mathrm{p}_{\mathrm{e}_{\mathrm{n}-1}}$ es la covarianza de ambiente permanente entre los caracteres, $\sigma_{\mathrm{e}_{1} \mathrm{e}_{2}}$ a $\sigma_{\mathrm{e}_{\mathrm{n}} \mathrm{e}_{\mathrm{n}-1}}$ es la covarianza residual entre los caracteres, $A$ es la matriz de relaciones, $I_{q}$ es la matriz identidad con orden $q$ ( $q$ es igual al número de madres) e $I_{n}$ es la matriz identidad de orden $n$ ( $n$ es igual al número de observaciones).

Los valores genéticos directos para el peso al nacimiento, al destete y a los 16 meses de edad (VG-PN, VG-PD y VG-P16m, respectivamente) fueron estimados por medio del mejor predictor lineal insesgado (BLUP) implementado en software WOMBAT (15). Luego haciendo uso de dichos valores genéticos, se llevó a cabo un análisis de componentes de principales mediante los paquetes FactoMineR (17) y factoextra (18) implementados en el software R. El objetivo de este análisis consistió en resumir la información contenida en los valores genéticos de cada carácter analizado en un conjunto más pequeño de nuevas dimensiones compuestas o variables que se pudieran visualizar gráficamente, llamadas componentes principales, y explorar como los cambios genéticos en un carácter podrían afectar a los otros, e indicar cuál de ellos podría usarse como criterio de selección $(8,9,11,13)$. Para ello, los valores genéticos directos fueron estandarizados (a media

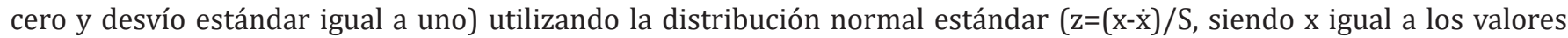
genéticos directos originales, x el valor promedio de dichos valores genéticos y $S$ que corresponde al desvío estándar respectivo). El número de componentes principales a considerar se eligió con base en el criterio de Kaiser (19).

Por último, con base en los componentes principales generados de los valores genéticos estandarizados se calculó un nuevo valor denominado puntaje del componente principal, el cual resultó de la suma de los valores genéticos estandarizados de cada carácter ponderado por su respectivo coeficiente estandarizado. Esto con el fin de proponer la construcción de un índice de selección para evaluar a los animales en busca de múltiples caracteres (13). Los coeficientes estandarizados en cada componente principal se obtuvieron usando la siguiente expresión:

$$
S S C_{i j}=\frac{\text { vectorpropio }_{i j}}{\sqrt{\text { valorpropio }_{j}}}
$$

dondeSSC $_{\mathrm{ij}}$ es el coeficiente estandarizado para los valores genéticos del i-ésimo carácter en el j-ésimo componente principal.

El puntaje del componente principal (índice) se calculó de la siguiente manera:

$$
P C_{j l}=\sum_{i=l}^{m} S S C_{i j} x V G_{i l}
$$

donde $\mathrm{PC}_{\mathrm{j} 1}$ es el puntaje del componente principal para el l-ésimo animal en el j-ésimo componente principal; $\mathrm{SSC}_{\mathrm{ij}}$ es el coeficiente de puntaje estandarizado para el valor genético del i-ésimo carácter en el j-ésimo componente principal; $\mathrm{VG}_{\mathrm{il}}$ es el valor genético estandarizado del i-ésimo carácter para el l-ésimo animal.

\section{RESULTADOS}

Los valores de heredabilidad directos obtenidos en este trabajo mediante análisis multivariado variaron entre $0.13 \pm 0.03$ para el peso al destete a $0.17 \pm 0.03$ para el peso al nacimiento. La heredabilidad materna estimada para el peso nacimiento, al destete y a los 16 meses de edad fueron de $0.09 \pm 0.03,0.33 \pm 0.04$ y $0.15 \pm 0.04$, respectivamente. En relación con la heredabilidad total (obtenida con base en la expresión, $\frac{\sigma_{a}^{2}+0.5 \sigma_{m}^{2}+1.5 \sigma_{a m}}{\sigma_{p}^{2}}$ siendo $\sigma_{\mathrm{a}}^{2}, \sigma_{\mathrm{m}}^{2} \mathrm{y} \sigma_{\mathrm{am}}$ los componentes de (co) varianza genética directa y materna presentados en la tabla 3), esta resultó en valores iguales a $0.18 \pm 0.04,0.16 \pm 0.04$ y $0.13 \pm 0.04$ para el peso al nacimiento, al destete y a los 16 meses de edad, respectivamente.

Las correlaciones genéticas entre los caracteres fueron de moderada a alta magnitud, con valores de $0.48 \pm 0.13$ entre el peso al nacimiento y al destete, de $0.25 \pm 0.17$ entre el peso al nacimiento y a los 16 meses de edad, y de $0.87 \pm 0.05$ entre el peso al destete y a los 16 meses de edad (Figura 1). 
Tabla 3. Componentes de varianza observados en los datos del análisis de caracteres productivos en bovinos Romosinuano.

\begin{tabular}{cccc}
\hline Componentes de varianza & Peso al nacimiento & Peso al destete & Peso a los 16 meses \\
\hline Genética directa $\left(\sigma_{\mathrm{p}}{ }^{2}\right)$ & 1.85 & 93.44 & 56.85 \\
Genética materna $\left(\sigma_{\mathrm{m}}{ }^{2}\right)$ & 0.95 & 237.00 & 139.20 \\
Covarianza directa y materna $\left(\sigma_{\mathrm{am}}\right)$ & -0.21 & -62.61 & -7.37 \\
Ambiente materno permanente $\left(\sigma_{\mathrm{c}}{ }^{2}\right)$ & ---- & 0.0016 & 709.19 \\
Residual $\left(\sigma_{\mathrm{e}}{ }^{2}\right)$ & 8.14 & 457.75 & 897.87 \\
*Fenotípica $\left(\sigma_{\mathrm{p}}{ }^{2}\right)$ & 10.73 & 725.58 & - \\
\hline
\end{tabular}

*La varianza fenotípica $\left(\sigma_{\mathrm{p}}{ }^{2}\right)$ fue igual a $\sigma_{\mathrm{a}}{ }^{2}+\sigma_{\mathrm{m}}{ }^{2}+\sigma_{\mathrm{c}}{ }^{2}+\sigma_{\mathrm{e}}{ }^{2}+\sigma_{\mathrm{am}}$; La heredabilidad directa $\left(\mathrm{h}_{\mathrm{a}}{ }^{2}\right)$ y materna $\left(\mathrm{h}_{\mathrm{m}}{ }^{2}\right)$ se calcularon con base en la expresión $\left(\sigma_{\mathrm{a}}^{2}\right) /\left(\sigma_{\mathrm{p}}{ }^{2}\right)$ y $\left(\sigma_{\mathrm{m}}{ }^{2}\right) /\left(\sigma_{\mathrm{p}}{ }^{2}\right)$, respectivamente; la correlación genética $\left(\mathrm{r}_{\mathrm{a}, 2}\right)$ se calculó con base en la expresión $\frac{4 \sigma_{a 12}}{\sqrt{4 \sigma_{a 1}^{2} \times \sigma_{a \alpha 2}^{2}}}$ ; la correlación ambiental $\left(\mathrm{r}_{\mathrm{e} 1,2}\right)$ se calculó con base en la expresión $\frac{4 \sigma_{e 1,2}}{\sqrt{4 \sigma_{e 1}^{2} x \sigma_{\sigma_{2}^{2}}^{2}}}$.

Fuente: Elaboración propia
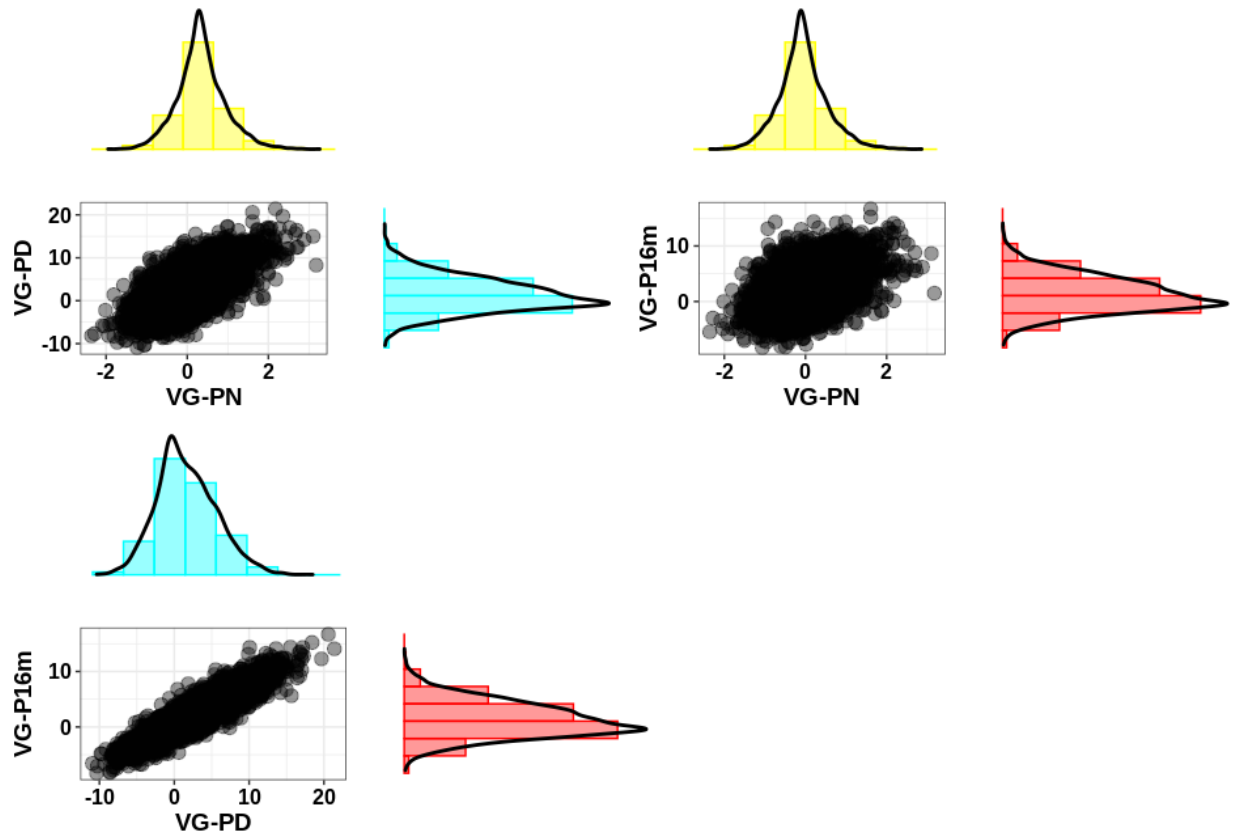

Figura 1. Graficas marginales de los valores genéticos directos (VG) del peso al nacimiento (PN), al destete (PD) y a los 16 meses de edad (P16m) en bovinos Romosinuano. Las gráficas de dispersión muestran la relación entre los valores genéticos estimados en dichos caracteres de crecimiento mientras que las gráficas de densidad muestran la distribución presentada por estos parámetros genéticos. Fuente: Elaboración propia

En el análisis de componentes principales (Tabla 4), el primer componente principal se eligió debido a que fue el único de los tres componentes resultantes en cumplir el criterio de Kaiser, que establece que solo los componentes principales con valores propios igual o mayor a la unidad son aquellos que explican la mayoría de la variación genética del conjunto de datos. Así mismo, el segundo componente principal se eligió debido a que fue el más cercano al valor umbral establecido por dicho criterio. En conjunto, ambos componentes principales elegidos explicaron aproximadamente el $97.99 \%$ del total de la variación de los valores genéticos directos estimados en los caracteres.

Tabla 4. Componentes principales y porcentaje de la variación explicados por estos en el análisis de caracteres productivos en bovinos Romosinuano.

\begin{tabular}{cccc}
\hline Componente principal & Valor propio & Variación(\%) & variación acumulada(\%) \\
\hline Primero & 2.30 & 77.94 & 77.94 \\
Segundo & 0.60 & 20.05 & 97.99 \\
Tercero & 0.06 & 2.01 & 100.00 \\
\hline
\end{tabular}

Fuente: Elaboración propia. 
La relación genética entre los valores genéticos directos (previamente estandarizados) para caracteres de crecimiento en bovinos Romosinuano se visualiza en la Figura 2. Este análisis permitió verificar cómo la selección basada en uno de dichos caracteres repercute sobre los otros. El valor genético de un carácter puede estar mayormente asociado con uno de los dos componentes principales cuando tiene un vector más grande y está más cerca de uno de los dos ejes. Los vectores en la misma dirección indican que los valores genéticos de los caracteres relacionados con estos vectores tienen correlaciones genéticas directamente proporcionales y los vectores en direcciones opuestas indican correlaciones genéticas inversas; producto de la ortogonalidad (o independencia) entre ambos componentes principales (primero y segundo) es posible seleccionar caracteres asociados con el primer componente principal sin alterar genéticamente los caracteres asociados con el segundo (8).

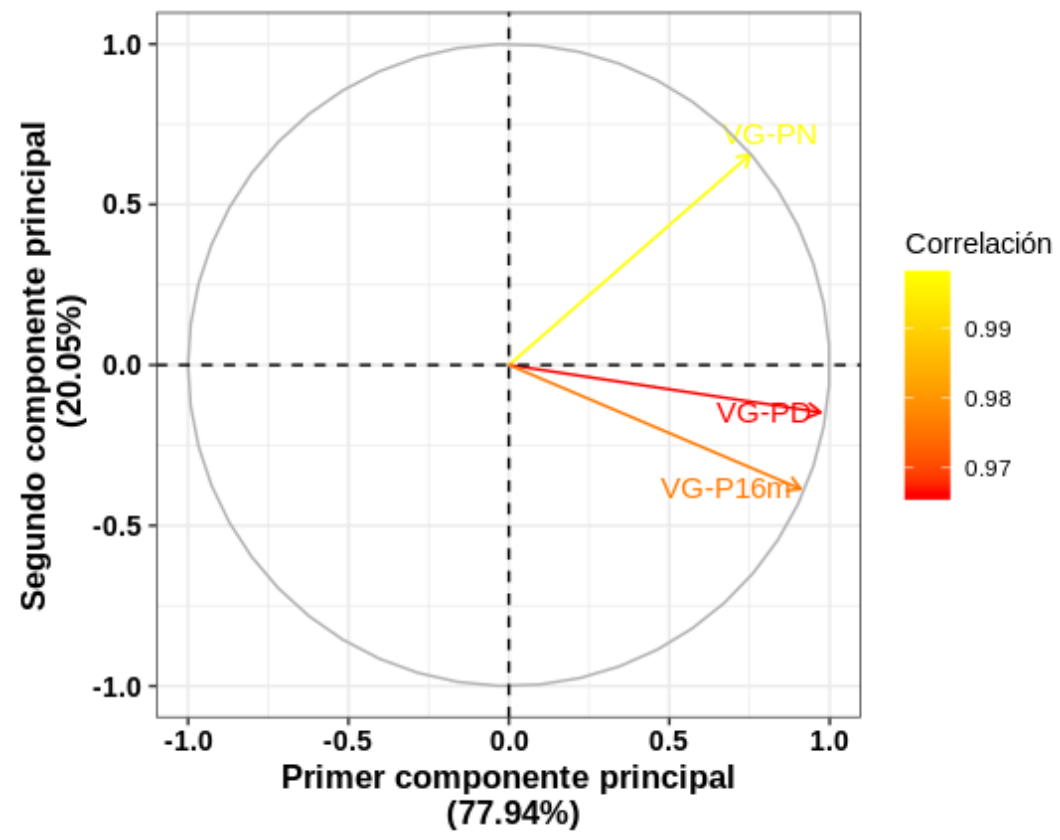

Figura 2. Gráfica de correlación de variables entre los valores genéticos directos (VG) para el peso al nacimiento (PN), al destete (PD) y a los 16 meses de edad (P16m) mediante análisis de componentes principales en bovinos Romosinuano. Se observa al lado derecho de la gráfica el valor de la relación entre las variables estandarizadas con el primer componente principal.

Fuente: Elaboración propia

Por consiguiente, y similar a los resultados de las correlaciones genéticas, el signo de la correlación entre los valores genéticos estimados para el peso al nacimiento (VG-PN), al destete (VG-PD) y a los 16 meses de edad (VG-P16m) con el primer componente principal, así como también la dirección del vector para cada valor genético, indican que los bovinos Romosinuano se pueden seleccionar con base en cualquiera de los caracteres de crecimiento para mejorar genéticamente los otros. Sobre la base de lo anterior, se recomendaría seleccionar en base al peso al nacimiento, puesto que al presentar la mayor heredabilidad (total) se tiene absoluta certeza de que las diferencias entre los bovinos al momento de realizado el pesaje son mayormente debido a los genes que poseen, y, en consecuencia, que serán transmitidos a su descendencia. No obstante, dada la correlación genética de magnitud moderada entre el peso al nacimiento con los pesos al destete y a los 16 meses de edad, la ganancia genética esperada para estos dos últimos caracteres mediante selección en base al peso al nacimiento será menor comparado con la ganancia genética esperada mediante selección directa (Tabla 5), por lo cual, al seleccionar los animales con base en el peso al nacimiento no se daría la posibilidad de promover ganancias genéticas rápidas en comparación con la selección en base a los otros caracteres.

La información sobre los coeficientes estandarizados en el primer componente principal de cada uno de los caracteres de crecimiento en bovinos Romosinuano se muestra en la Tabla 6. Utilizando los coeficientes se generó un índice (puntaje del primer componente principal, $\mathrm{PC}_{1}$ ) para la selección de los mejores bovinos Romosinuano en relación con los valores genéticos previamente estandarizados (VG) del peso al nacimiento (PN), al destete (PD) y a los 16 meses de edad (P16m), el cual se presenta a continuación:

$\mathrm{PC}_{1}=(-0.32 x V G-P N)+(-0.41 x V G-P D)+(-0.39 x V G-P 16 m)$ 
Tabla 5. Progreso genético para caracteres de crecimiento en bovinos Romosinuano, correlación genética entre cada carácter con el peso al nacimiento, y respuesta correlacionada y eficiencia relativa de selección de dichos caracteres realizando selección en base al peso al nacimiento.

\begin{tabular}{|c|c|c|c|c|}
\hline & \multicolumn{4}{|c|}{${ }^{*}$ Progreso genético $(\Delta \mathrm{G})$ Correlación genética $(\mathrm{ra}){ }^{* *}$ Respuesta correlacionada $(\mathrm{RC}){ }^{* * *}$ Eficiencia relativa de selección $(\mathrm{ER})$} \\
\hline Peso al nacimiento & 0.59 & 1.00 & ---- & ---- \\
\hline Peso al destete & 4.31 & 0.48 & 2.17 & 50.34 \\
\hline Peso a los 16 meses de edad & 3.89 & 0.25 & 1.13 & 29.05 \\
\hline
\end{tabular}

*El progreso genético $(\Delta \mathrm{G})$ fue igual h2xixopa donde h2 es la heredabilidad para cada carácter, $i$ es la intensidad de selección para cada carácter en unidades de desviación estándar (equivalente a 1.00) y $\sigma_{\mathrm{p}}$ es el desvió fenotípico para cada carácter. ${ }^{* *}$ La respuesta correlacionada ( $R C$ ) fue igual a $r_{a} x h_{1} x h_{2} x i x \sigma_{2}$ donde $r_{a}$ es la correlación genética entre cada carácter de crecimiento con el peso al nacimiento, $h_{1}$ es la raíz cuadrada de la heredabilidad del peso al nacimiento, $h_{2}$ es la raíz cuadrada de la heredabilidad de cada carácter, $i$ es la intensidad de selección (equivalente a 1.00) y $\sigma_{2}$ es el desvió fenotípico del peso al destete y a los 16 meses de edad. ${ }^{* * *}$ La eficiencia relativa de selección $(E R)$ fue igual a $R C \div \Delta G \times 100$ donde $R C$ es la respuesta correlacionada y $\Delta G$ es el progreso genético.

Fuente: Elaboración propia

Tabla 6. Vectores propios y coeficientes estandarizados (SSC) en el primer componente principal para la construcción de un índice de selección para caracteres de crecimiento en bovinos Romosinuano.

\begin{tabular}{ccc}
\hline Carácter & Vector propio $\left(\mathbf{P C}_{\mathbf{1}}\right)$ & ${ }^{*} \mathbf{S S C}\left(\mathbf{P C}_{\mathbf{1}}\right)$ \\
\hline Peso al nacimiento & -0.49 & -0.32 \\
Peso al destete & -0.63 & -0.41 \\
Peso a los 16 meses de edad & -0.59 & -0.39 \\
\hline
\end{tabular}

*Cuanto mayor sea el valor absoluto del SSC, mayor será la importancia relativa del valor genético estandarizado en el primer componente principal.

Fuente: Elaboración propia

El análisis de componentes principales permitió la construcción de un índice de selección mediante la combinación lineal entre los valores genéticos estandarizados disponibles en el conjunto de datos, teniendo en cuenta tanto el valor propio del componente principal, como el vector propio del carácter en cada componente principal, los cuales son medidas de variabilidad. De esta manera, los caracteres de crecimiento en bovinos Romosinuano podrán ser incluidos en procesos de selección, aun cuando debido a sus estimaciones de heredabilidad (total) de magnitud baja, es posible que tengan escaso uso en dichos procesos.

\section{DISCUSIÓN}

En general, las estimaciones de heredabilidad total para todos los caracteres en este estudio fueron inferiores a las reportados en la literatura. Esta discrepancia pudo ser debido a diferencias genéticas entre poblaciones, a los modelos estadísticos utilizados y al sistema de manejo (efecto ambiental); son iguales en PN y mayores en PD y P16 meses a los obtenidos por Ossa et al (4) quienes reportan valores de $0.17 \pm 0.03,0.09 \pm 0.02$ y $0.06 \pm 0.03$ respectivamente. Al respecto, Cardellino y Rovira (20) reportan que la heredabilidad es una valor relativo y no absoluto, en el sentido de que es propio de la población que sirvió para su estimación, no obstante, aluden que dicho valor se puede extrapolar a otras poblaciones con estructura genética similar y expuestas a un medio ambiente común.

De acuerdo con Tramonte et al (11), los caracteres medidos hasta el destete pueden estar influenciados por efectos maternos. En este estudio se pudo evidenciar que existen también caracteres que al ser medidos después de este evento pueden estar sujetos a dicho efecto, en este caso el peso a los 16 meses de edad. Sin embargo, el efecto materno puede ser menor en este último carácter en comparación con otros caracteres como el peso al destete, debido a que a los 16 meses de edad las variaciones del potencial de producción de las vacas pueden tener menor impacto en el desarrollo de sus crías (21).

En la literatura al igual que lo observado en este estudio, se reporta correlaciones genéticas de magnitud media y alta entre el peso al nacimiento, al destete y a los 16 meses de edad $(22,23,24,25)$, lo que indica, según Ossa (7), que dichos caracteres están determinados, en gran proporción, por la acción de los mismos genes (efecto pleiotrópico de los genes sobre estos caracteres). 
Además de conocer en qué medida los caracteres están influenciados por los mismos genes, es importante saber el efecto conjunto de sus genes, una vez la selección por uno de ellos puede resultar o no en una ganancia genética para el otro dependiendo de la dirección de la relación (20). En este aspecto, dada la relación positiva de la relación genética entre los caracteres de crecimiento en bovinos Romosinuano (Figura 1), es posible indicar que la selección con base en uno de ellos podría conllevar al mejoramiento genético de los otros. No obstante, y contrario a los resultados obtenido por López et al (26) para caracteres de producción de leche en bovinos Lucerna, la selección en base al carácter identificado como criterio de selección no conllevaría a una ganancia genética de los otros caracteres, dado que, si bien dicha relación genética es positiva, la misma es de magnitud moderada.

Las correlaciones fenotípicas entre los caracteres de crecimiento en bovinos Romosinuano, fueron de 0.35 entre los pesos al nacimiento y al destete, de 0.25 entre los pesos al nacimiento y a los 16 meses de edad, y de 0.59 entre los pesos al destete y a los 16 meses de edad. Sin embargo, se recomienda que la asociación entre los caracteres sea observada mediante la correlación genética y no la fenotípica, puesto que es sabido que aun cuando exista una correlación fenotípica positiva entre los caracteres, la selección por uno de ellos no resulta necesariamente en una ganancia genética para el otro, dada que esta es una correlación de origen tanto genético como ambiental (20).

Son escasos los trabajos encontrados donde se haya empleado el análisis de componentes principales en las ciencias animales. Savegnago et al (9) en un estudio de una población de gallinas usaron el análisis de componentes principales para identificar caracteres que podrían usarse como criterios de selección para mejorar aspectos relacionados con la producción de huevos; Tramonte et al (11) haciendo uso de datos productivos y reproductivos de la hembra bovina Guzerat, con base en el valor de heredabilidad, la correlación genética positiva entre caracteres y mediante el uso de componentes principales llegaron a la conclusión de que la selección de animales podría hacerse en función de unos pocos caracteres en lugar de todos ellos, mencionando además que las ganancias genéticas de cada carácter estarían en la misma dirección de aquel elegido como criterio de selección.

De igual forma, a partir del análisis de componentes principales es posible seleccionar animales con base en los puntajes generados por dichos componentes en lugar de los valores genéticos para los tres caracteres por separado. En este sentido, Ruales y Manrique (12) determinaron un mayor progreso genético (aproximadamente un 56\%) mediante la selección basada en un índice propuesto a partir de componentes principales en comparación con el método de selección individual.

Del presente trabajo se pueden sacar las siguientes conclusiones, El peso al nacimiento puede ser usado como criterio de selección para mejorar los caracteres de peso al destete y a los 16 meses de edad en bovinos Romosinuano dado su valor de heredabilidad y relación con dichos caracteres. Así, la ganancia genética del peso al nacimiento mediante procesos de selección se atribuirá de la misma manera en los otros caracteres estudiados.

En el caso complejo del análisis multivariado, el análisis mediante componentes principales puede usarse como una buena alternativa para simplificar las interpretaciones de todas las correlaciones genéticas juntas en dos componentes principales.

Es posible construir índices de selección basados en componentes principales y así mejorar a partir de su uso los caracteres de crecimiento en bovinos Romosinuano a pesar de las bajas heredabilidades de estos.

\section{Conflicto de intereses}

Todos los autores realizaron aportes significativos al documento, están de acuerdo con su publicación y manifiestan que no existen conflictos de interés en este estudio. La información no es confidencial ni estratégica.

\section{Agradecimientos}

Los autores reconocen y agradecen a todos los integrantes del equipo del proyecto: "Generación de recomendaciones técnicas basadas en el confort y el bienestar animal para mejorar la fertilidad de los bovinos en el trópico", ejecutado por la Corporación Colombiana de Investigación Agropecuaria-AGROSAVIA, Financiado por el MADR y al SBGNAA. 


\section{REFERENCIAS}

1. Domínguez-Viveros J, Urbina-Valenzuela AR, Palacios-Espinoza A, Callejas-Juárez N, Ortega-Gutiérrez JÁ, EspinozaVillavicencio JL, et al. Crecimiento y primer empadre en vacas Siboney. Ecosist Recur Agropec. 2019; 6(17):383-389. https://doi.org/10.19136/era.a6n17.1786

2. Vergara GOD, Flórez MJM, Hernández PMJ, Yaguna GCJ, Manco JC, Barrios RTE, et al. Efectos raciales, de heterosis y parámetros genéticos para peso al nacer en una población multirracial de ganado de carne en Colombia. LLRD. 2014; 26(3). http://www.lrrd.org/lrrd26/3/verg26058.html

3. Ramírez Valverde R, Martínez Rocha RE, Núñez Domínguez R, García Muñiz JG. Genetic parameters and trends of growth traits for Romosinuano cattle in Mexico. Nova Scientia. 2018; 10(21):310-325. https://doi.org/10.21640/ ns.v10i21.1595

4. Ossa G, López J, Santana-Rodriguez M, Garcés J. Heredabilidad y tendencias genéticas para caracteres del crecimiento en bovinos criollos Romosinuano. Arch Latinoam de Prod Anim. 2021; 29(3-4):143-154.

5. Bezerra junior J, Silva F, Hongyu K, Couto A, Medeiros R, Araújo F, et al. Multivariate analysis of milk yield, lactation length, and calving interval in female buffaloes. Rev Colomb Cienc Pecu. 2018; 31(2):103-109. https://doi. org/10.17533/udea.rccp.v31n2a03

6. Martínez C, Manrique C, Elzo M. La evaluación genética de bovinos: una percepción histórica. Rev Colomb Cienc Pecu. 2012; 25:293-311. https://revistas.udea.edu.co/index.php/rccp/article/view/324760

7. Ossa, G. Mejoramiento genético animal aplicado a los sistemas de producción de carne. Universidad Nacional de Colombia. Medellín, 2017.

8. Nascimento G, Savegnago R, Chud T, Ledur M, Figueiredo E, Munari D. Genetic parameter estimates and principal component analysis on performance and carcass traits of a terminal pig sire line. Acta Agric Scand A Anim Sci. 2014; 64(3):137-144, https://doi.org/10.1080/09064702.2014.950322

9. Savegnago R, Caetano S, Ramos S, Nascimento G, Schmidt G, Ledur M, et al. Estimates of genetic parameters, and cluster and principal components analyses of breeding values related to egg production traits in a White Leghorn population. Poult Sci. 2011; 90:2174-2188. https://doi.org/10.3382/ps.2011-01474

10. Venturini G, Savegnago R, Nunes B, Ledur M, Schmidt G, El Faro L, et al. Genetic parameters and principal component analysis for egg production from White Leghorn hens. Poult Sci. 2013; 92:2283-2289. https://doi.org/10.3382/ ps.2013-03123

11. Tramonte NC, Grupioni NV, Stafuzza NB, Guidolin DG, Savegnago RP, Bezerra (et al). Genetic parameters, genetic trends, and principal component analysis for productive and reproductive traits of Guzera beef cattle. R Bras Zootec. 2019; 48:e20180034. https://doi.org/10.1590/rbz4820180034

12. Ruales F, Manrique C. Uso del análisis de componentes principales para construir un índice tipo producción en ganado Romosinuano (Bos taurus). Rev Colomb Cienc Pecu. 2007; 20:124-128. http://www.scielo.org.co/scielo. php?script=sci arttext\&pid=S0120-06902007000200004.

13. Buzanskas M, Savegnago R, Grossi D, Venturini G, Queiroz S, Silva L, et al. Genetic parameter estimates and principal component analysis of breeding values of reproduction and growth traits in female Canchim cattle. Reprod Fertil Dev. 2013; 25(5):775-781. https://doi.org/10.1071/rd12132.

14. R Core Team. [En línea]. R: A language and environment for statistical computing. 2019. http://www.R-project.org/

15. Meyer K. WOMBAT, a program for mixed model analyses by restricted maximum likelihood. [En línea]. 2016. http:// didgeridoo.une.edu.au/km/wombat.php 
16. Wilson A, Réale D, Clements M, Morrissey M, Postma E, Walling C, et al. An ecologist's guide to the animal model. J Anim Ecol. 2010; 79:13-26. https://doi.org/10.1111/j.1365-2656.2009.01639.x

17. Husson F, Josse J, Le S, Mazet J. [En línea]. FactoMineR: multivariate exploratory data analysis and data mining. 2020. https://github.com/cran/FactoMineR

18. Kassambara A, Mundt F. Factoextra: extract and visualize the results of multivariate data analyses. 2019. https:// github.com/kassambara/factoextra.

19. Kaiser HF. The application of electronic computers to factor analysis. Educ Psychol Meas 1960; 20:141-151. https:// doi.org/10.1177\%2F001316446002000116

20. Cardellino R, Rovira J. Mejoramiento genético animal. Montevideo (Uruguay): Hemisferio Sur. 1987.

21. Martínez R, Pérez J. Parámetros y tendencias genéticas para características de crecimiento en el ganado criollo colombiano Romosinuano. Revista Corpoica - Ciencia y Tecnología Agropecuaria. 2006; 7(1):25-32. https://doi. org/10.21930/rcta.vol7 num1 art:56

22. Gallego J, Martínez R, Moreno F. Índice de consanguinidad y caracterización fenotípica y genética de la raza bovina criolla Blanco Orejinegro. Revista Corpoica - Ciencia y Tecnología Agropecuaria. 2006; 7(1):16-24. https://doi. org/10.21930/rcta.vol7 num1 art:55

23. Martínez R, Pérez J, Herazo T. Evaluación fenotípica y genética para características de crecimiento en la raza criolla colombiana Costeño con Cuernos. Revista Corpoica - Ciencia y Tecnología Agropecuaria. 2006; 7(2):12-20. https:// doi.org/10.21930/rcta.vol7 num2_art:65

24. Vergara 0, Pérez J, Noriega J, Cencio L, Martínez N. Análisis multicarácter para estimar componentes de (co)varianza en características de crecimiento en bovinos costeño con cuernos. Livestock research for rural development. 2018; 30(9):30161. http://www.lrrd.org/lrrd30/9/overg30161.html

25. Bedoya C, Alzate J, Ángel J, Escobar C, Calvo S. Evaluación genética para características de peso en ganado Brahman comercial. Rev MVZ Córdoba. 2019; 24(2):7225-7230. https://doi.org/10.21897/rmvz.1662

26. López J, Quijano J, González L. Estimación de parámetros genéticos para producción de leche en el día de control y a los 305 días en primeras lactancias de vacas Lucerna. LRRD. 2019; 31(11):31179. http://www.lrrd.org/lrrd31/11/ luggo31179.html 